\title{
Renal Allograft Biopsy Findings in Live-related Renal Transplant Recipients
}

\author{
Syed Munib, Tanveer Ahmed, Raheel Ahmed and Najam-ud-Din \\ Department of Nephrology and Renal Transplantation, Institute of Kidney Diseases, Peshawar, Pakistan
}

\begin{abstract}
Objective: To determine the pattern of histopathology in living-related, kidney transplant recipients (KTRs) from a transplant centre in Khyber Pakhtunkhwa (KPK), Pakistan.

Study Design: Descriptive, observational study.

Place and Duration of Study: Institute of Kidney Diseases, Peshawar, from August 2008 to July 2018.

Methodology: A retrospective review of graft biopsy reports and clinical charts from living-related, kidney transplant recipients was carried out. Allograft biopsies were done for graft dysfunction with no apparent cause. The biopsy pathology was classified according to updated Banff classifications. The descriptive statistics were used to tabulate the results.

Results: Out of the 55 biopsies, $51(92.73 \%)$ were from males with mean age of $34.35 \pm 9.40$ years. Out of 52 percutaneous biopsies, $10(19.23 \%)$ belonged to the normal category. Category 2 (borderline rejection) and 3 (acute/active cellular rejection) were seen in three (5.7\%) and one (1.9\%) cases, respectively. Interstitial fibrosis/tubular atrophy (Banff Category 5) was observed in 18 (34.62\%) cases. Banff Category 6 (others) was seen in 19 (36.5\%) cases, in which calcineurin inhibitors (CNI) toxicity was commonest (17 [89.4\%] of 19 cases). Mixed lesions were found in 19 (36.5\%) cases. Out of the 19 mixed category cases, 12 (63.16\%) showed both Category 3 and Category 5 changes with most of the cases showing mild to moderate IF/TA; while one case had severe IF/TA. Three graft nephrectomies were done, one each for recurrent oxalosis, nephroblastoma and fungal infection.

Conclusion: Among the studied specimens, mixed lesions were the predominant findings, followed by others (mostly CNI toxicity) and IFTA categories. The frequency of acute/active rejections was low and that of chronic changes higher, in keeping with delayed biopsies.
\end{abstract}

Key Words: Allograft biopsy, Graft dysfunction, Rejection, Kidney.

How to cite this article: Munib S, Ahmed T, Ahmed R, NUD. Renal Allograft Biopsy Findings in Live-related Renal Transplant Recipients. J Coll Physicians Surg Pak 2021; 31(02):197-201.

\section{INTRODUCTION}

Chronic kidney disease (CKD) is one of the major public health problems worldwide and a significant cause of morbidity and mortality, contributing to global economic burden. ${ }^{1} \mathrm{~A}$ report published by US Renal Data System (USRDS) reveals that there were 120,688 new cases of end-stage renal disease (ESRD) reported in 2014 with an annual rise of $1.1 \% .{ }^{2}$ In Pakistan, it has been estimated that the incidence of ESRD is 100 per million population. ${ }^{3}$ The majority of patients who are started on hemodialysis, either die or stop treatment because of financial constraints within the first three months and less than $2 \%$ of patients are started on ambulatory peritoneal dialysis. Even though renal transplantation is the economical option, only about $4-5 \%$ of all patients with ESRD succeed ingetting a transplant. ${ }^{4}$

Correspondence to: Dr. Syed Munib, Department of Nephrology and Renal Transplantation, Institute of Kidney Diseases, Peshawar, Pakistan

E-mail: munibsyed@gmail.com

Received: June 20, 2020; Revised: August 19, 2020;

Accepted: September 16, 2020

DOI: https://doi.org/10.29271/jcpsp.2021.02.197
Renal transplantation is the preferred treatment for ESRD patients all over the world. ${ }^{5}$ In recent times, the success rate of renal transplantation has improved considerably. ${ }^{6}$ According to the recent data, 20 -year-graft survival could be expected in $60 \%$ of kidney transplant recipients. ${ }^{7}$ The short-term kidney transplant outcomes have improved dramatically during the last few decades due to better surgical techniques, overall improved medical care, early diagnosis and treatment of infections, and last but not least, the advancements in transplant immunology. ${ }^{6-8}$

In spite of the above advances, renal allograft dysfunction is still common after transplantation. The major causes of graft dysfunction are acute or chronic rejection, calcineurin inhibitor (CNI) toxicity, infections and recurrence of/de novo primary kidney disease. The above causes of renal allograft malfunction require different therapeutic approaches; thus, an accurate diagnosis is a pre-requisite for correct management, which can only be ascertained at present through renal allograft biopsy. However, scarce data is available in published literature solely on the causes of graft malfunction in a living-related kidney transplant programme. ${ }^{6-9}$ Renal transplant biopsy (RTB) is well established as the gold standard procedure to determine the etiology of allograft dysfunction. ${ }^{5-10}$ According to a study 
published previously, biopsy findings change the clinical diagnosis in an average of $36 \%$ of patients and therapy in $59 \% .{ }^{11}$ Renal transplant pathology is a complex and rapidly evolving field and an analysis of the pathological lesions sheds light on the prevailing practices and emerging trends in clinical transplantation field. ${ }^{8}$

This study was aimed to determine the pattern of histopathological lesions causing renal graft dysfunction as detected on kidney graft biopsies of living-related kidney transplant recipients (KTRs) from a transplant centre in Khyber Pakhtunkhwa (KPK), Pakistan and to relate these results to those reported previously in literature.

\section{METHODOLOGY}

A retrospective cross-sectional study of renal allograft biopsies of renal allograft recipients was conducted over a period of 10 years from August 2008 to July 2018 at the Institute of Kidney Diseases, Peshawar, Pakistan. Data items including demographics of recipients and renal allograft biopsy findings were collected from reviews of renal allograft biopsy reports. The biopsies were done when there was graft dysfunction without obvious cause, i.e., an increase in serum creatinine of $\geq 30 \%$ from the baseline. The second main indication was proteinuria. ${ }^{12-15} \mathrm{~A}$ very conservative biopsy policy was employed to arrive at the correct diagnosis by non-invasive techniques. Two needle cores of kidney graft tissue were obtained with automated biopsy gun under real-time ultrasound guidance. In cases in which there was clinical suspicion for antibody-mediated rejection (ABMR), a third core was attempted for immunofluorescence, $C 4 d$, and ultrastructural study. ${ }^{16}$ The benefits and risks of the biopsy procedure were fully explained to the patients and their families, and they signed a written consent form. The biopsies were then sent to a specialised renal pathology laboratory, in formalin-containing sterile container for further processing and reporting. The biopsy lesions were reported and classified according to Banff 2017 updated classification of kidney graft pathology. ${ }^{13}$

All patients received standard induction therapy with Interleukin-2 (IL-2) receptor blocker Basiliximab (Simulect), two doses of $20 \mathrm{mg}$ intravenously, each on day 0 ( 2 hours pre-transplant) and on $4^{\text {th }}$ day post-transplant. Patients received standard maintenance immunosuppressive therapy in standard dosage, i.e., one of the CNIs (Cyclosporine [CSA] or tacrolimus (TAC), one of the anti-proliferative agents (Azathioprine [AZA]/Mycophenolate mofetil (MMF), and/or one of mammalian target of rapamycin (mTOR) inhibitors (everolimus or sirolimus) and steroids.

The research was conducted according to the ethical standards of the Declaration of Helsinki.

Statistical analysis was carried out using the Statistical Package for the Social Sciences (SPSS) software, version 21.0 (IBM Corp, New York, NY, USA). Descriptive statistics of mean \pm standard deviation (SD) were used for normally distributed continuous variables, such as age and clinical and laboratory data. For categorical data, such as biopsy diagnoses, numbers (percentages) were used.

\section{RESULTS}

A total of 55 renal allograft biopsies were obtained over the period of 10 years from August 2008 to July 2018. Out of these biopsies, 51 (92.73\%) were from males, while $4(7.27 \%)$ were from females. Their mean age was $34.35 \pm 9.40$ years, ranging from 19 to 60 years. Majority of patients ( $n=24,43.63 \%$ ) belonged to the age group of 31-42 years. The mean age of donors was $38.45 \pm 7.86$ years, ranging from 20 to 65 years. Regarding donor relationships with the recipients, the majority of donors were siblings (27: $49.09 \%)$ followed by parents (17: $30.91 \%$ ) and others (11: $20 \%$ ). The biopsies were done at a mean post-transplant duration of $18.37 \pm 9.51$ months, ranging from 4 days to 4.5 years. The mean serum creatinine at the time of biopsy was $3.34 \pm 1.64 \mathrm{mg} / \mathrm{dl}$, ranging from 1.6 to $8.3 \mathrm{mg} / \mathrm{dl}$.

Table I: Categorisation of percutaneous renal allograft biopsy $(n=52)$ findings according to BanffClassification 2017.

\begin{tabular}{|l|c|c|}
\hline Banff categories & Number (n) & $\%$ \\
\hline Category 1 (Normal) & 10 & 19.23 \\
\hline Category 2 (ABMR) & 1 & 1.92 \\
\hline Category 3 (Borderline changes) & 3 & 5.77 \\
\hline Category 4 (TCMR) & 1 & 1.92 \\
\hline Category 5 (IF/TA) & 18 & 34.62 \\
Mild IFTA & 4 & 22.22 \\
Moderate IFTA & 8 & 44.44 \\
Severe IFTA & 6 & 33.33 \\
\hline Category 6 (Others) & 19 & 36.54 \\
Calcineurin inhibitors (CNI) toxicity & 17 & 89.4 \\
Recurrent/de novo GN & 1 & 5.3 \\
Infection & 1 & 5.3 \\
\hline Total & 52 & 100 \\
\hline ABMR: Antibody-mediated rejection; IFTA: Interstitial fibrosis/tubular atrophy; \\
TCMR: T-cell-mediated rejection.
\end{tabular}

Of the remaining three cases, two cases underwent graft nephrectomies, one $(1.82 \%)$ case had recurrent oxalosis, while one (1.82\%) was diagnosed with nephroblastoma. One (1.82\%) biopsy showed only fungal hyphae in completely infarcted parenchyma; and no viable graft tissue was identified and graft had to be removed.The majority of allograft biopsies (84\%) were adequate, according to Banff criteria. The biopsy findings in 52 percutaneous renal allograft biopsies with clear-cut histological results were categorised according to updated Banff schema and are shown in Table I. It was observed that 10 (19.23\%) cases belonged to Banff Category 1 (normal). Category 2 (borderline rejection) and three (acute/active cellular rejection) were seen in three $(5.7 \%)$ and one $(1.9 \%)$ cases, respectively. Banff Category 5 of interstitial fibrosis/tubular atrophy (IFTA) with varying degrees of severity (mild, moderate or severe) was observed in $18(34.62 \%)$ cases. Banff Category 6 (others) was seen in $19(36.5 \%)$ cases, mostly seen in conjunction with other categories, in which calcineurin inhibitors (CNI) toxicity was commonest (17 [89.4\%] of 19 cases). Mixed lesions were seen in 19 (36.5\%) cases. Out of 19 mixed category cases, $12(63.16 \%)$ showed both Category 3 and Category 5 changes with most of the cases showing mild to moderate IF/TA, while one case had severeIF/TA. 


\section{DISCUSSION}

A number of efforts have been made to standardise the histological criteria for the diagnosis and classification of acute rejection to allow comparisons of efficacy of different treatment modalities. ${ }^{17}$ Various classification systems that have been introduced include the Banff classification system, which was first published in full form in $1993,{ }^{18}$ and the cooperative clinical trials in transplantation (CCTT) modification of it. ${ }^{19}$ Theseclassification systems concerted upon inflammation involving arteries and tubules but differed on the minimum threshold of inflammation and typing/grading of rejection. The Banff 93-95 and the CCTT systems were both merged into Banff 97 working classification. ${ }^{20}$ According to 1997 Banff classification, increased histologic severity has strong correlation with poor response to treatment and shortened allograft survival. ${ }^{21}$ The Banff 97 system has been revised several times. ${ }^{18,20-22}$

This study provides significant contribution to the existing scant data, especially in the setting of the living-related kidney transplant programme from Pakistan. The demographic characteristics of the recipients are almost in line to that reported in a previous study from Pakistan. ${ }^{8}$ Male preponderance is in concordance with the prior studies conducted in Pakistan, ${ }^{8}$ and other South-Asian countries like India ${ }^{23}$ and Nepal. ${ }^{24}$ The mean age of recipients observed in this study was $34.34 \pm 9.39$ years with most of the patients, $43.63 \%$, falling in age group of $31-42$ years. This age distribution is also consistent with previously conducted study from Nepal. ${ }^{24}$

In this study, the authors witnessed that $16.36 \%$ of the biopsies belonged to Banff Category 1 (normal) i.e., the biopsies showed no evidence of any abnormality, which is comparable to that found by Koshy et al; ${ }^{23}$ while in contrast to studies from Nepal and Pakistan. ${ }^{8,24}$ Another study done in Macedonia observed $31 \%$ of cases belonging to this category. ${ }^{7}$ Moreover, these findings are in agreement with the study done in Belgium by Naesens et al..$^{25}$ The varying results may indicate the varying biopsy policies and less strict indication criteria employed in some studies. ${ }^{8}$ Ideally, this category should be the least common finding in the biopsies, if thorough work-up is done prior to performing the biopsy. There can be many causes for this finding in the biopsy in the setting of indicated biopsies, such as sampling error, missed pre- or post-graft causes of dysfunction, etc.

Acute antibody-mediated rejection (ABMR) can occur in recipients who develop rising titers of anti-donor antibodies immediately after transplant surgery or are pre-sensitised and transplanted without prior desensitisation. Acute ABMR usually develops 1-3 weeks after transplantation, commonly in sensitised patients, but can develop at any time post-transplant. ${ }^{21}$ Chronic ABMR, in contrast to acute ABMR, usually presents years after transplantation.

It evolves through numerous stages over many months to years. ${ }^{22}$ It may be difficult to make a distinction between acute ABMR and severe acute T-cell mediated rejection (TCMR), and sometimes the two disease processes may also overlap. Moreover, in up to $25 \%$ of cases of graft dysfunction attributed, at least in part, to ABMR, the biopsy features are indicative of only TCMR or acute tubular injury. ${ }^{16}$ It is vital to diagnose and report $A B M R$, if possible, given that it is often unresponsive to therapy targeting acute TCMR and, unless successfully treated, often leads to renal graft loss. ${ }^{21,25}$ In this study, one case each of isolated acute ABMR (Category 2), one of acute TCMR (Category 4), and one of chronic active ABMR, were observed. This observation is comparable to some previous studies, especially those involving live-related transplants, ${ }^{7,8}$ but much lower from a large cohort from Europe. ${ }^{25}$ It is important to recognise and reportchronic ABMR, as it is amenable to treatment. ${ }^{13,22}$

Borderline changes (Banff Category 3) were found, as sole lesions, in $3(5.77 \%)$ cases, which is similar to study from Macedonia, ${ }^{7}$ India, ${ }^{23}$ and Nepal; ${ }^{24}$ and in contrast to the study from Europe.$^{25}$ However, a significant number of these lesions were found in association with chronic changes (Banff Category 5 of IFTA), and if the numbers are corrected for the borderline changes and IFTA, the percentage gets even slightly higher than the previously published European study. ${ }^{25}$

Banff Category 4 of TCMR lesions were found, as sole lesions, in one case and combined with ABMR in another one case. In addition, five TCMR cases were found in association with chronic changes in this study. Thus, total number of TCMR cases were seven $(13.46 \%)$ in our series, which is compatible with recent series from around the world. ${ }^{25}$

IFTA, no evidence of any specific etiology (NOS) (Banff Category 5 ) was found in $18(34.62 \%)$ of total cases and is one of the commonest causes of graft dysfunction in this study, which was, in majority of cases, without any specific etiology as published previously. ${ }^{8,22,23}$ But in studies from Macedonia ${ }^{7}$ and Nepal, ${ }^{24}$ a lower prevalence of IFTA was observed. This may be due, in part,

to the fact that the graft biopsies were performed late on the patients of this study and that IF/TA takes longer than other causes of renal allograft dysfunction to develop; and it has been documented in previously published studies that IFTA is more prevalentafterfirst post-transplantyear. ${ }^{8,25}$

While analysing the results of this study, it is of note that almost one-third of total cases showed more than one pattern of histopathological lesions or category of rejection and were included in mixed category. This has also been reflected by previously published studies. ${ }^{7,25}$ However, studies from Nepal and Pakistan showed lower prevalence of mixed lesions. ${ }^{8,24}$ This finding also points to the complexity of renal transplant pathology and in part may be affected by varying biopsy policies.

The "other" (Banff Category 6) causes of graft dysfunction were seen in conjunction with the other various Banff categories in nearly one-fifth of cases and it can be conferred that calcineurin inhibitors (CNI) toxicity was one of the leading causes of graft dysfunction in the renal transplant recipients. The toxicity of the agents may be caused by high, within range or even low concen- 
trations of the culprit in the blood. ${ }^{20}$ We observed that almost $90 \%$ of all cases, that were included in "others" category, showed CNI toxicity on renal graft biopsy, usually as an arterial hyalinosis grade 2 or 3 , which is in keeping with studies done in past from Pakistan ${ }^{8}$ and our neighboring countries like India $^{23}$ and Nepal. ${ }^{24}$

There are some limitations in the study. It is a retrospective study. Although it spans a period of 10 years but number of biopsies is not high. This small number can be attributed to low number of patients opting for renal transplantation in our region and the conservative biopsy policy. The authors also did not take into account the clinical picture of patients and correlate them to their biopsy findings in the current study.

\section{CONCLUSION}

The study showed that the mixed lesions were the predominant findings, followed by others (mostly CNI toxicity) and IFTA categories. The frequency of acute/active rejections was low and that of chronic changes higher, in keeping with delayed biopsies.

\section{ETHICAL APPROVAL:}

Ethical approval was granted by Hospital Ethical Review Committee (Ref. No. 600 dated 02-07-2018) prior to initiation of the research work.

\section{PATIENTS' CONSENT:}

Written informed consent was taken from all patients before obtaining biopsies and for publishing the data.

\section{CONFLICT OF INTEREST:}

Authors declared no conflict of interest.

\section{AUTHORS' CONTRIBUTION:}

SM, TA, RA: Conception, study designing and statistical analysis, and supervision.

TA, RA, ND: Data collection and manuscript writing.

SM, TA, RA: Critical review and final approval of manuscript.

\section{REFERENCES}

1. Schieppati A, Remuzzi G. Chronic renal diseases as a public health problem: Epidemiology, social, and economic implications. Kidney Int Suppl 2005; (98):S7-S10. doi: 10.1111/j.1523-1755.2005.09801.x.

2. Saran R, Robinson B, Abbott KC, Agodoa LY, Albertus P, Ayanian J, et al. US renal data system 2016 annual data report: Epidemiology of kidney disease in the United States. Am J Kidney Dis 2017; 69(3 Suppl 1):A7-A8. doi: 10.1053/j.ajkd.2016.12.004.

3. Ullah K, Butt G, Masroor I, Kanwal K, Kifayat F. Epidemiology of chronic kidney disease in a Pakistani population. Saudi J Kidney Dis Transplant 2015; 26(6):1307-10. doi: 10.4103/ 1319-2442.168694.

4. Rizvi SA, Naqvi SA, Zafar MN, Hussain Z, Hashmi A, Hussain $M$, et al. A renal transplantation model for developing countries. Am J Transplant 2011; 11(11):2302-7. doi: 10.1111/j.1600-6143.2011.03712.x.

5. Wang Z, Yang H, Liu X, Zhang J, Han Z, Tao J, et al. Role of
$\mathrm{B}$ and $\mathrm{T}$ lymphocyte attenuator in renal transplant recipients with biopsy-proven acute rejection. Med Sci Monit 2018; 24:387-96. doi: 10.12659/msm.905752.

6. Han Y, Guo H, Cai M, Xiao L, Wang Q, Xu X, et al. Renal graft biopsy assists diagnosis and treatment of renal allograft dysfunction after kidney transplantation: A report of 106 cases. Int J Clin Exp Med 2015; 8(3):4703-7.

7. Severova-Andreevska G, Grcevska L, Petrushevska G, Cakalaroski K, Sikole A, Stojceva-Taneva O, et al. The spectrum of histopathological changes in the renal allograft - a 12 months protocol biopsy study. Open Access Maced J Med Sci 2018; 6(4):606-12. doi: 10.3889/oamjms.2018.162.

8. Kazi Jl, Mubarak M. Biopsy findings in renal allograft dysfunction in a live related renal transplant program. J Transplant Technol Res 2012; 2:108. doi:10.4172/21610991.1000108.

9. Puntambekar A, Parameswaran S, Nachiappa RG. Evaluation of clinico-pathological spectrum in renal allograft biopsies at JIPMER. J Kidney 2017; 03(03). doi: 10.4172/ 2472-1220.1000149.

10. Schmid A. Percutaneous renal transplant biopsy: Is the safety profile adequate for short-term postprocedure monitoring? Transpl Int 2016; 29(2):165-6. doi: 10.1111/ tri. 12713.

11. Broecker V, Mengel M. The significance of histological diagnosis in renal allograft biopsies in 2014. Transpl Int 2015; 28(2):136-43. doi: 10.1111/tri.12446.

12. Serón D, Anaya F, Marcén R, del Moral RG, Martul EV, Alarcón $A$, et al. Guidelines for indicating, obtaining, processing, and evaluating kidney transplant biopsies. Nefrologia 2008; 4:385-96.

13. Haas M, Loupy A, Lefaucheur C, Roufosse C, Glotz D, Seron $D$, et al. The banff 2017 kidney meeting report: Revised diagnostic criteria for chronic active $T$ cell-mediated rejection, antibody-mediated rejection, and prospects for integrative endpoints for next-generation clinical trials. $\mathrm{Am} J$ Transplant 2018; 18(2):293-307. doi:10.1111/ajt.14625.

14. Loupy A, Haas M, Solez K, Racusen L, Glotz D, Seron D, et al. The banff 2015 kidney meeting report: Current challenges in rejection classification and prospects for adopting molecular pathology. Am J Transplant 2017; 17(1):28-41. doi: 10.1111/ajt.14107.

15. Tsai SF, Chen $\mathrm{CH}$, Shu KH, Cheng CH, Yu TM, Chuang YW, et al. Current safety of renal allograft biopsy with indication in adult recipients: An observational study. Medicine (Baltimore) 2016; 95(6):e2816. doi: 10.1097/MD. 0000000000002816.

16. Mauiyyedi S, Crespo M, Collins AB, Schneeberger EE, Pascual MA, Saidman SL, et al. Acute humoral rejection in kidney transplantation: II. Morphology, immunopathology, and pathologic classification. J Am Soc Nephrol 2002; 13(3):779-87.

17. Furness PN, Taub N. Convergence of European renal transplant pathology assessment procedures $P$. International variation in the interpretation of renal transplant biopsies: Report of the CERTPAP project. Kidney Int 2001; 60(5):1998-2012. doi: 10.1046/j.1523-1755.2001. 00030.x.

18. Solez K, Axelsen RA, Benediktsson H, Burdick JF, Cohen AH, 
Colvin RB, et al. International standardisation of criteria for the histologic diagnosis of renal allograft rejection: The banff working classification of kidney transplant pathology. Kidney Int 1993; 44(2):411-22. doi: 10.1038/ki.1993.259.

19. Colvin RB, Cohen AH, Saiontz C, Bonsib S, Buick M, Burke B, et al. Evaluation of pathologic criteria for acute renal allograft rejection: Reproducibility, sensitivity, and clinical correlation. J Am Soc Nephrol 1997; 8(12):1930-41.

20. Racusen LC, Solez K, Colvin RB, Bonsib SM, Castro MC, Cavallo $\mathrm{T}$, et al. The Banff 97 working classification of renal allograft pathology. Kidney Int 1999; 55(2):713-23. doi: 10.1046/j.1523-1755.1999.00299.x.

21. Racusen LC, Colvin RB, Solez K, Mihatsch MJ, Halloran PF, Campbell PM, et al. Antibody-mediated rejection criteria an addition to the Banff 97 classification of renal allograft rejection. Am J Transplant 2003; 3(6):708-14. doi: 10.1034/j.1600-6143.2003.00072.x.
22. Haas M, Sis B, Racusen LC, Solez K, Glotz D, Colvin RB, et al. Banff 2013 meeting report: inclusion of c4d-negative antibody-mediated rejection and antibody-associated arterial lesions. Am J Transplant 2014; 14(2):272-83. doi: 10.1111/ajt.12590.

23. Koshy PJ, Tripathy A, Vijayan M, Nair S, Yuvaraj A, Natarajan $G$, et al. A multicentre study of the spectrum of histopathological changes in renal allograft biopsies over a period of nine years from South India. Immunopathol Persa 2017; 3(1):e05.

24. Aryal G, Shah D. Histopathological evaluation of renal allograft biopsies in Nepal: Interpretation and significance. J Pathol Nepal 2012; 2(3):172-9. doi.org/10.3126/jpn. v2i3.6016

25. Naesens M, Kuypers DR, De Vusser K, Evenepoel P, Claes K, Bammens $B$, et al. The histology of kidney transplant failure: A long-term follow-up study. Transplantation 2014; 98(4):427-35. doi: 10.1097/TP.0000000000000183. 